\title{
Regression Analysis between Multiple Osteoporotic Spine Fracture and Osteoporotic Fracture Risk Factors
}

\author{
Jaewon Lee, Ye-Soo Park
}

Department of Orthopedic Surgery, Guri Hospital, Hanyang University

College of Medicine, Guri city, Gyunggi-do, Korea 


\section{Introduction}

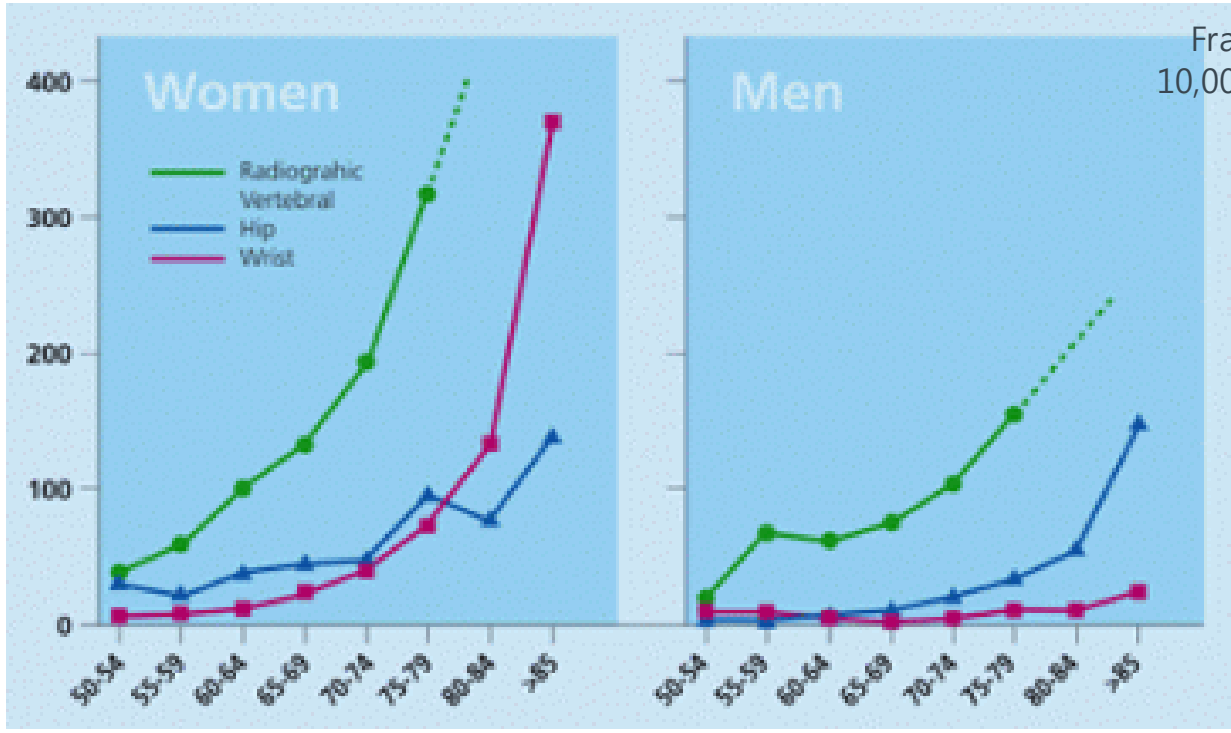

Fractures per

$\rightarrow$-Spine $-t$-Wrist

Hip
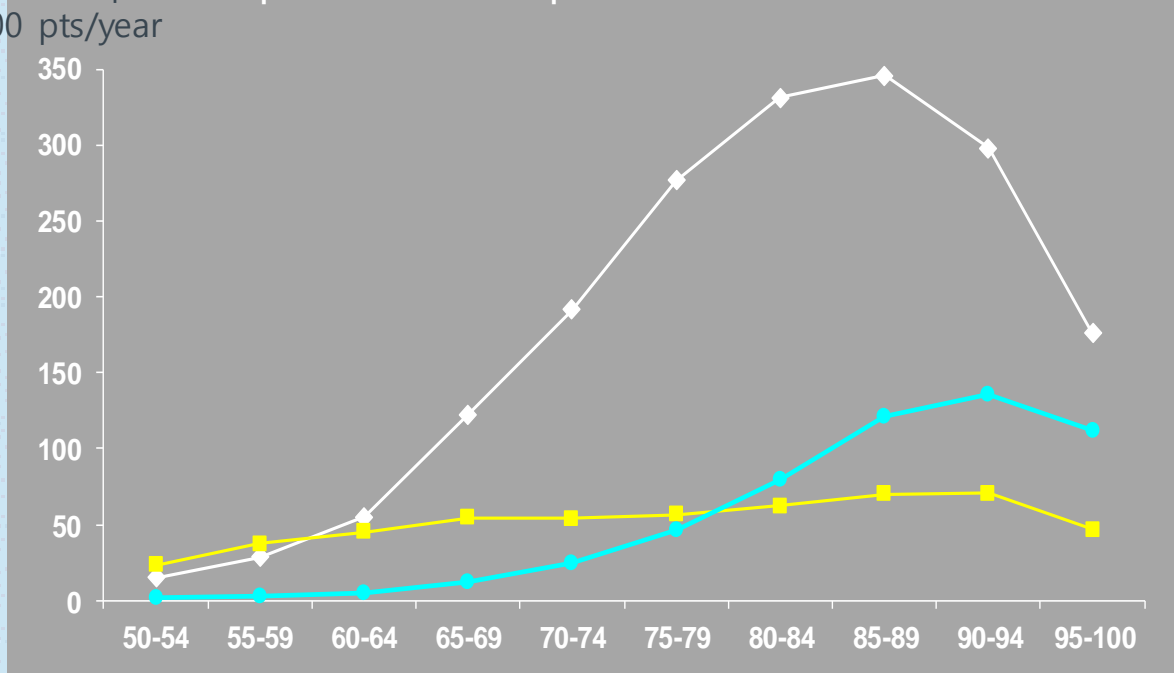

Age, years

Sambrook et al., Lancet., 2006 Jang et al., HIRA Research report., 2009 


\section{Introduction}

- Severity of osteoporotic spine fracture $1 \Longrightarrow$ Subsequent fracture 1

Delmas et al., Bone., 2003

- All low-trauma osteoporotic fractures were associated with increased mortality risk in the first 5 years.

- Subsequent fracture was associated with $\times 3 \sim 4$ increased mortality risk for an additional 5 years.

\section{What's the difference between}

general osteoporotic spine fracture

and

severe multiple osteoporotic spine fracture? 


\section{Materials \& Methods}

- From January 2014 to May 2015

- Inclusion

- Patients who checked the bone marrow density (BMD) using a dual-energy x-ray absorptiometry(DEXA) (Delphi-W, Hologic, Waltham, MA, USA)

- Informed consent agreement

- Exclusion

- Spine fracture due to high-energy trauma that is defined according to the fracture morphology and patient's history.

- Past history of taking drugs that may affect bone metabolism.

- Past history of spine surgery. 


\section{Materials \& Methods}

527 Patients

126 Exclusion criteria

401 Patients

232 Lack of data

\section{Patients}

\section{Group 1} (No OSF)

60
Group 2

(1 OSF)

71
Group 3 ( $\geq 3$ OSF) 40

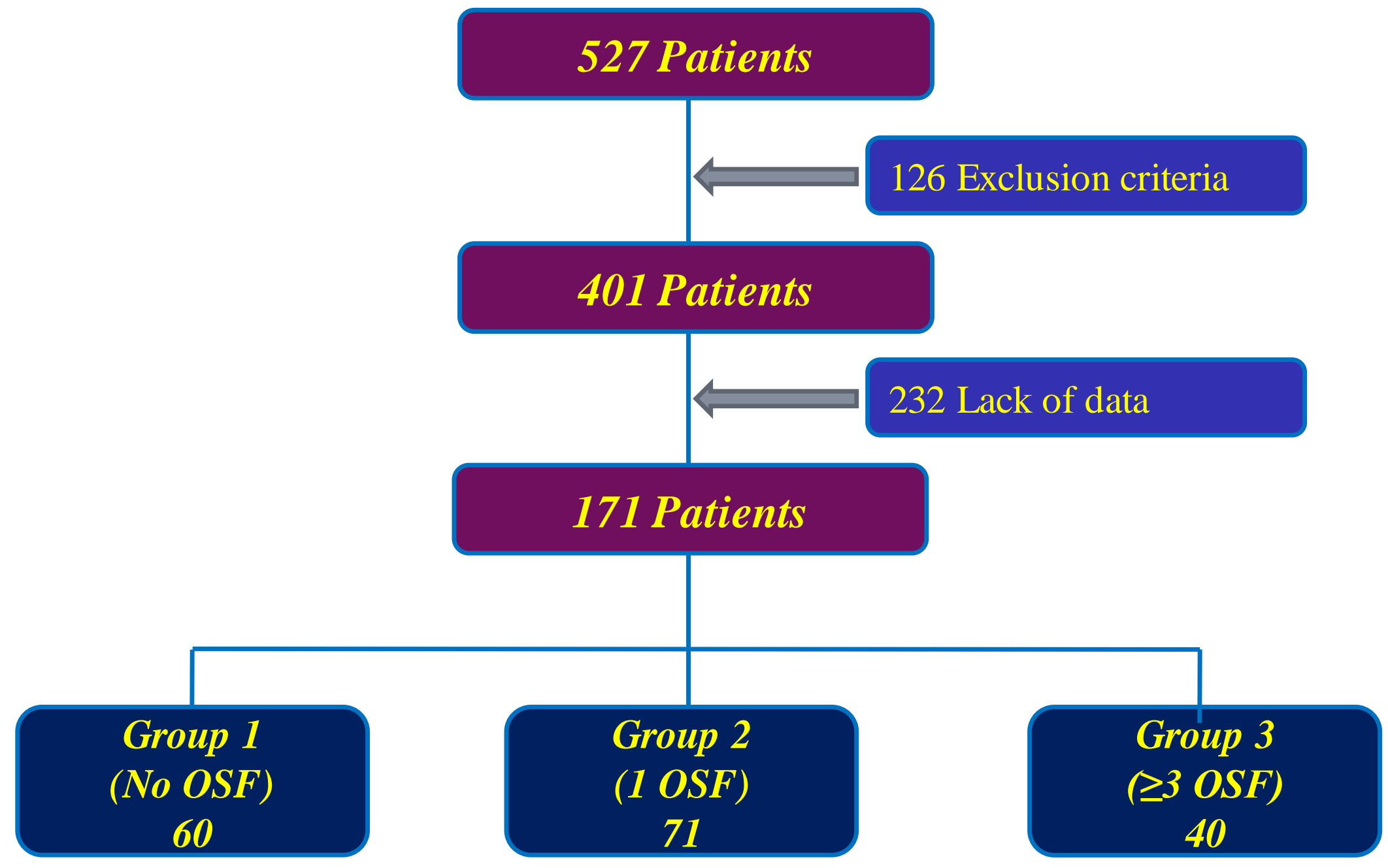




\section{Materials \& Methods}

- Age

- Body mass index (BMI)

- Bone marrow density (BMD)

- Presence of rheumatoid arthritis (RA)

- Use of steroid (Steroid)

- Osteoporosis treatment (Osteo. Tx.)

- Smoking

- Drinking

- Serum 25(OH) vitamin $D_{3}\left(\right.$ Vit. $\left.D_{3}\right)$

- Past history of osteoporotic fracture ( Past Osteo. Fx.) 


\section{Results}

\begin{tabular}{|c|c|c|c|c|}
\hline Mean \pm SD & Non-OSF $(\mathrm{N}=60)$ & 1 OSF (N=71) & $>3$ OSF $(\mathrm{N}=40)$ & Total (N=171) \\
\hline Age & $70.40 \pm 5.64$ & $72.61 \pm 9.01$ & $70.68 \pm 12.92$ & $71.38 \pm 9.16$ \\
\hline BMI & $24.38 \pm 3.72$ & $22.04 \pm 2.60$ & $20.48 \pm 2.34$ & $22.50 \pm 3.34$ \\
\hline BMD & $-2.03 \pm 0.97$ & $-2.96 \pm 1.12$ & $-3.59 \pm 0.82$ & $-2.78 \pm 1.17$ \\
\hline Vit. $D_{3}$ & $31.67 \pm 18.94$ & $23.70 \pm 11.11$ & $15.87 \pm 4.64$ & $24.67 \pm 14.71$ \\
\hline $\operatorname{Sex}(M: F)$ & 22 : $38(63.3 \%)$ & $10: 61(85.9 \%)$ & $3: 37$ (92.5\%) & $35: 136(79.5 \%)$ \\
\hline RA (Non RA: RA) & $56: 4(6.7 \%)$ & $60: 11(15.5 \%)$ & 32 : 8 (20.0\%) & $148: 23(13.5 \%)$ \\
\hline $\begin{array}{l}\text { Steroid } \\
\text { (No history of steroid therapy: } \\
\text { history of steroid therapy) }\end{array}$ & 57 : $3(5.0 \%)$ & 62 : $9(12.7 \%)$ & 34 : 6 (15.0\%) & $153: 18(10.5 \%)$ \\
\hline $\begin{array}{c}\text { Osteo. TX. } \\
\text { (No historyof osteoporosis } \\
\text { treatment: historyof } \\
\text { osteoporosis treatment) }\end{array}$ & 52 : $8(13.3 \%)$ & $55: 16(22.5 \%)$ & 33 : 7 (17.5\%) & $140: 31(18.1 \%)$ \\
\hline $\begin{array}{c}\text { Smoking } \\
\text { (Non-smoker:Smoker) }\end{array}$ & $58: 2(3.3 \%)$ & 64 : $7(9.8 \%)$ & 34 : 6 (15.0\%) & $156: 15(8.8 \%)$ \\
\hline $\begin{array}{l}\text { Drinking } \\
\text { (No drinker: } 3 \text { or more } \\
\text { alcoholic beverages a day) }\end{array}$ & $56: 4(6.7 \%)$ & $64: 7(9.8 \%)$ & 35 : 5 (12.5\%) & $155: 16(9.4 \%)$ \\
\hline $\begin{array}{l}\text { Past Osteo. Fx. } \\
\text { (No previous osteoporotic } \\
\text { fracture history: Previous } \\
\text { osteoporotic fracture history) }\end{array}$ & 55 : $5(8.3 \%)$ & $53: 18(25.4 \%)$ & $25: 15(37.5 \%)$ & $133: 38(22.2 \%)$ \\
\hline
\end{tabular}


; Non-OSF vs. 1 OSF // Non-OSF vs. >3 OSF

\begin{tabular}{|c|c|c|c|c|c|c|c|c|}
\hline \multirow{2}{*}{ Non-OSF } & $\begin{array}{l}\text { Odds } \\
\text { ratio }\end{array}$ & \multicolumn{2}{|c|}{$95 \% \mathrm{Cl}$ for OR } & $p$-value & $\begin{array}{l}\text { Odds } \\
\text { ratio }\end{array}$ & \multicolumn{2}{|c|}{$95 \% \mathrm{Cl}$ for OR } & $p$-value \\
\hline & \multicolumn{4}{|c|}{1 OVF } & \multicolumn{4}{|c|}{3 OVF } \\
\hline Age & 1.028 & 0.989 & 1.069 & 0.166 & 1.003 & 0.962 & 1.046 & 0.888 \\
\hline BMI & 0.770 & 0.673 & 0.881 & 0.000 & 0.618 & 0.516 & 0.740 & 0.000 \\
\hline BMD & 0.421 & 0.288 & 0.618 & 0.000 & 0.203 & 0.119 & 0.348 & 0.000 \\
\hline Vit. $D_{3}$ & 0.963 & 0.937 & 0.989 & 0.006 & 0.861 & 0.808 & 0.917 & 0.000 \\
\hline Sex & 3.532 & 1.509 & 8.264 & 0.004 & 7.140 & 1.969 & 25.898 & 0.003 \\
\hline RA & 2.567 & 0.772 & 8.529 & 0.124 & 3.500 & 0.977 & 12.543 & 0.54 \\
\hline Steroid & 2.758 & 0.711 & 10.695 & 0.142 & 3.353 & 0.787 & 14.287 & 0.102 \\
\hline Osteo. Tx. & 1.891 & 0.746 & 4.790 & 0.179 & 1.379 & 0.457 & 4.160 & 0.569 \\
\hline Smoking & 3.172 & 0.633 & 15.886 & 0.160 & 5.118 & 0.978 & 26.790 & 0.053 \\
\hline Drinking & 1.531 & 0.426 & 5.506 & 0.514 & 2.000 & 0.503 & 7.957 & 0.325 \\
\hline Past osteo. Fx. & 3.736 & 1.294 & 10.785 & 0.015 & 6.600 & 2.160 & 20.169 & 0.001 \\
\hline
\end{tabular}




\begin{tabular}{|c|c|c|c|c|c|c|c|c|}
\hline \multirow{2}{*}{ Non-OVF } & $\begin{array}{l}\text { Odds } \\
\text { ratio }\end{array}$ & \multicolumn{2}{|c|}{$95 \% \mathrm{Cl}$ for OR } & $p$-value & $\begin{array}{l}\text { Odds } \\
\text { ratio }\end{array}$ & \multicolumn{2}{|c|}{$95 \% \mathrm{Cl}$ for OR } & $p$-value \\
\hline & \multicolumn{4}{|c|}{1 OVF } & \multicolumn{4}{|c|}{3 OVF } \\
\hline Age & 1.031 & 0.977 & 1.088 & 0.260 & 1.006 & 0.944 & 1.073 & 0.846 \\
\hline BMI & 0.847 & 0.722 & 0.994 & 0.042 & 0.641 & 0.493 & 0.832 & 0.001 \\
\hline BMD & 0.524 & 0.311 & 0.883 & 0.015 & 0.174 & 0.074 & 0.408 & 0.000 \\
\hline Vit. $D_{3}$ & 0.953 & 0.921 & 0.987 & 0.007 & 0.779 & 0.705 & 0.859 & 0.000 \\
\hline Sex & 1.615 & 0.539 & 4.836 & 0.392 & 2.122 & 0.335 & 13.462 & 0.425 \\
\hline RA & 1.806 & 0.418 & 7.808 & 0.428 & 2.148 & 0.339 & 13.630 & 0.417 \\
\hline Steroid & 2.994 & 0.500 & 17.951 & 0.230 & 8.583 & 0.871 & 84.618 & 0.066 \\
\hline Osteo. Tx. & 1.472 & 0.470 & 4.614 & 0.507 & 1.174 & 0.257 & 5.361 & 0.836 \\
\hline Smoking & 0.722 & 0.112 & 4.640 & 0.731 & 0.317 & 0.036 & 2.813 & 0.303 \\
\hline Drinking & 1.281 & 0.283 & 5.803 & 0.748 & 2.011 & 0.285 & 14.163 & 0.483 \\
\hline Past Osteo. Fx. & 1.197 & 0.328 & 4.364 & 0.785 & 0.810 & 0.156 & 4.206 & 0.802 \\
\hline
\end{tabular}




\section{Conclusions}

- Risk factors of multiple osteoporotic spine fracture has no difference between osteoporotic spine fracture happened 1 level.

- But, multiple osteoporotic spine fracture has lower BMI, BMD, Vit. D3 than osteoporotic spine fracture happened 1 level.

- Females, and having more past history of osteporotic fracture might have more chance to get the multiple osteoporotic spine fracture. 